\title{
AN APPROXIMATE ANALYTIC SOLUTION OF A PARTICULAR BOUNDARY VALUE PROBLEM
}

\author{
UGUR TANRIVER and ARAVINDA KAR
}

(Received 8 January 2001)

\begin{abstract}
This note is concerned with the three-dimensional quasi-steady-state heat conduction equation subject to certain boundary conditions in the whole $x^{\prime} y^{\prime}$-plane and finite in $z^{\prime}$-direction. This type of boundary value problem arises in laser welding process. The solution to this problem can be represented by an integral using Fourier analysis. This integral is approximated to obtain a simple analytic expression for the temperature distribution.
\end{abstract}

2000 Mathematics Subject Classification. 35C05, 41A46, 44A15.

1. Mathematical formulation and analysis. The governing equation for the nondimensional temperature distribution is given by [2]

$$
T_{x x}+T_{y y}+T_{z z}=-(P e) T_{x}
$$

The boundary conditions for the temperature are

$$
\begin{gathered}
T \longrightarrow 0 \quad \text { as } x \longrightarrow \pm \infty, y \rightarrow \pm \infty \\
\left.T_{z}\right|_{z=0}=-\frac{2 A P}{\pi r_{0} k_{w}\left(T_{m}-T_{0}\right)} \exp \left[-2\left(x^{2}+y^{2}\right)\right]+N u_{t} T \\
\left.T_{z}\right|_{z=d}=-N u_{b} T
\end{gathered}
$$

where $A, P, r_{0}, k_{w}, T_{m}, T_{0}, P e, N u_{t}$, and $N u_{b}$ are all constants defined in [2]. Equation (1.1) can be simplified by defining

$$
T=T_{1} \exp \left(-\frac{P e}{2} x\right)
$$

which transforms (1.1) into the following form:

$$
T_{1 x x}+T_{1 y y}+T_{1 z z}-\left(\frac{P e^{2}}{4}\right) T_{1}=0
$$

and, the boundary conditions are now expressed as

$$
\begin{gathered}
T_{1} \longrightarrow 0 \text { as } x \rightarrow \pm \infty, y \rightarrow \pm \infty, \\
\left.T_{1 z}\right|_{z=0}=-\frac{2 A P}{\pi r_{0} k_{w}\left(T_{m}-T_{0}\right)} \exp \left[-2\left(x^{2}+y^{2}\right)\right]+N u_{t} T_{1}, \\
\left.T_{1 z}\right|_{z=d}=-N u_{b} T_{1} .
\end{gathered}
$$


The solution to the boundary value problem was obtained earlier [2]. The solution is given in dimensional form

$$
T^{\prime}\left(x^{\prime}, y^{\prime}, z^{\prime}\right)=T_{0}+\frac{A P \exp [-P e / 2(x-P e / 16)]}{2 \pi r_{0} k_{w}} \int_{0}^{\infty} R \exp \left(-\frac{R^{2}}{8}\right) \Phi(R) J_{0}(r R) d R,
$$

where $J_{0}(\beta)$ is the Bessel function of order zero, $x=x^{\prime} / r_{0}, y=y^{\prime} / r_{0}, z=z^{\prime} / r_{0}$,

$$
\begin{gathered}
\Phi(R)=\frac{M \cosh [M(d-z)]+N u_{b} \sinh [M(d-z)]}{\left(M^{2}+N u_{t} N u_{b}\right) \sinh (M d)+M\left(N u_{t}+N u_{b}\right) \cosh (M d)}, \\
M=\sqrt{R^{2}+\frac{P e^{2}}{4}},
\end{gathered}
$$

and, $R$ is the distance in the Fourier space (dual variable) defined as $R=\sqrt{p^{2}+q^{2}}$ and $r$ is the distance in the real $x y$-plane defined as $r=\sqrt{x^{2}+y^{2}}$.

To approximate the integral in (1.6), consider the special case $N u_{b}=N u_{t}=0$. For this case (1.7) becomes

$$
\Phi(R)=\frac{\cosh [M(d-z)]}{M \sinh (M d)}
$$

For large values of $M$ which is $R \rightarrow \infty$, (1.8) can be approximated as

$$
\Phi(R) \approx \frac{\exp (-M z)+\exp [-M(2 d-z)]}{M} .
$$

Therefore, the integral in (1.6) becomes

$$
I \approx \int_{0}^{\infty} R \exp \left(-\frac{R^{2}}{8}\right) \frac{\exp (-M z)+\exp [-M(2 d-z)]}{M} J_{0}(r R) d R .
$$

Approximation of the following type of integral is necessary in general:

$$
I_{A}=\int_{0}^{\infty} R \exp \left(-a R^{2}\right) \frac{\exp \left(-c \sqrt{R^{2}+b^{2}}\right)}{\sqrt{R^{2}+b^{2}}} J_{0}(r R) d R,
$$

where $a, b$, and $c$ are positive constants. One can rewrite (1.11) as

$$
I_{A}=\exp \left(a b^{2}\right) \int_{0}^{\infty} R \exp \left(-a \sqrt{R^{2}+b^{2}} \sqrt{R^{2}+b^{2}}\right) \frac{\exp \left(-c \sqrt{R^{2}+b^{2}}\right)}{\sqrt{R^{2}+b^{2}}} J_{0}(r R) d R .
$$

Expanding one of the repeated terms under the square root sign in the first exponential term in a Taylor series about $R=0$ and retaining the first term, one obtains

$$
I_{A} \approx \exp \left(a b^{2}\right) \int_{0}^{\infty} R \frac{\exp \left[-(c+a b) \sqrt{R^{2}+b^{2}}\right]}{\sqrt{R^{2}+b^{2}}} J_{0}(r R) d R .
$$


Using the Hankel transform [1], (1.13) can be evaluated as

$$
I_{A}=\exp \left(a b^{2}\right) \frac{\exp \left[-b \sqrt{r^{2}+(c+a b)^{2}}\right]}{\sqrt{r^{2}+(c+a b)^{2}}} .
$$

Now the approximated dimensional temperature distribution can be obtained from (1.14)

$$
\begin{aligned}
T^{\prime}\left(x^{\prime}, y^{\prime}, z^{\prime}\right) \approx & T_{0}+\frac{A P \exp [-P e / 2(x-P e / 16)]}{2 \pi r_{0} k_{w}} \exp \left(\frac{P e^{2}}{32}\right) \\
& \times\left[\frac{\exp \left(-P e / 2 \sqrt{r^{2}+(z+P e / 16)^{2}}\right)}{\sqrt{r^{2}+(z+P e / 16)^{2}}}\right. \\
& \left.+\frac{\exp \left(-P e / 2 \sqrt{r^{2}+(2 d-z+P e / 16)^{2}}\right)}{\sqrt{r^{2}+(2 d-z+P e / 16)^{2}}}\right] .
\end{aligned}
$$

2. Results and discussion. Figures 2.1, 2.2, and 2.3 show the numerical and approximate temperature distributions along the $z^{\prime}$-axis for various values of the radial distance in the real physical space such as, $r=0,0.45$ and $0.75 \mathrm{~mm}$ with laser power $50 \mathrm{~W}$, absorptivity $36 \%$, laser beam radius $0.15 \mathrm{~mm}$ and scanning speed $10 \mathrm{~mm} / \mathrm{s}$. As expected, the temperature profiles below $z^{\prime}=50 \mu \mathrm{m}$ are not in good agreement for the case of $r=0$ because the approximation is accurate for larger values of $r$. Figure 2.1 depicts that the two temperature values get closer to each other for $z^{\prime}$ values lying in the interval $[50,200]$. As shown in Figures 2.2 and 2.4, the difference between the temperature values decreases along $z^{\prime}$-axis for larger values of $r$. Figure 2.4 shows the comparison between the numerical and approximate temperature values along the $y^{\prime}$-axis for $x^{\prime}=0,0.15 \mathrm{~mm}$. For $y^{\prime}$ values up to $80 \mu \mathrm{m}$, there is a discrepancy between the two temperature values for $x^{\prime}=0$. However, it is good for $x^{\prime}=0.15 \mathrm{~mm}$. In addition, all the temperature values are in very good agreement for $y^{\prime}$ values lying in the interval $[160,450]$.

The approximate temperature values can also be improved for small and large values of $r$. One of the expression under the square root sign of exponent is approximated as

$$
\sqrt{R^{2}+b^{2}} \approx b \frac{r+A^{\prime}}{r+B^{\prime}}
$$

where $A^{\prime}$ and $B^{\prime}$ are parameters to be determined numerically. In addition, a multiplicative factor $C^{\prime}$ is introduced for integral in (1.12). In this case the result is very much improved for $r=0$ as shown in Figure 2.5. As $r$ increases, the difference between the numerical and approximate temperature values becomes smaller. These cases are depicted in Figures 2.6 and 2.7. Figure 2.8 shows very good agreement between the two temperatures for $x^{\prime}=0$ and all $y^{\prime}$ values compared to Figure 2.4. 


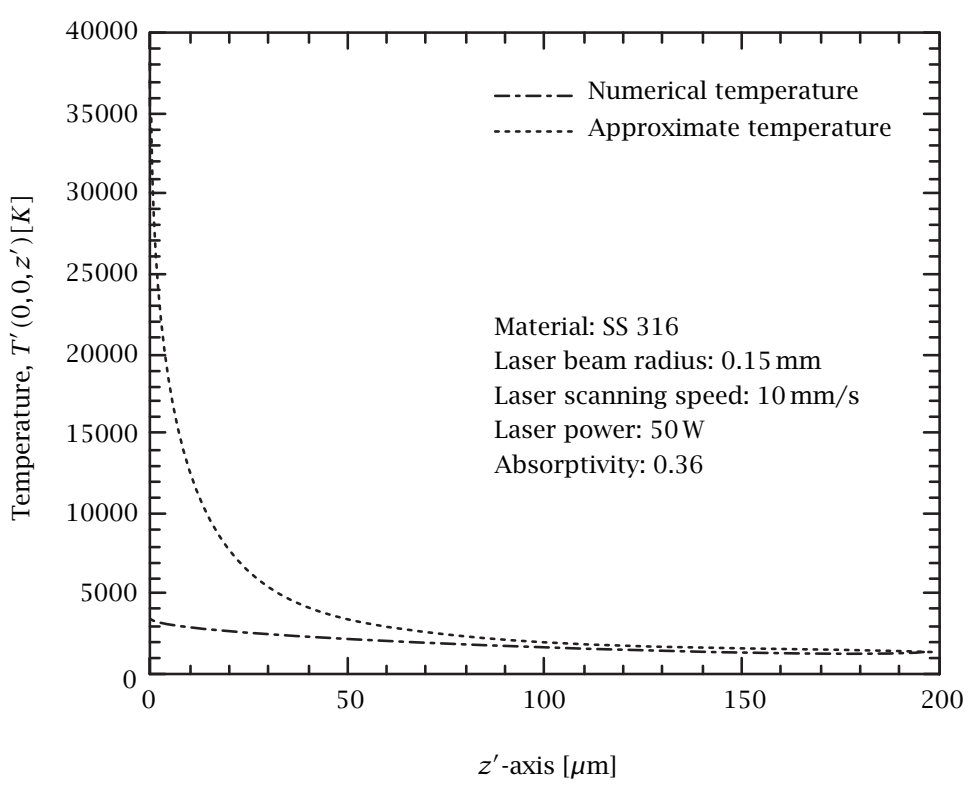

FIGURE 2.1. Comparison between the numerical and approximate temperatures $(r=0$ case).

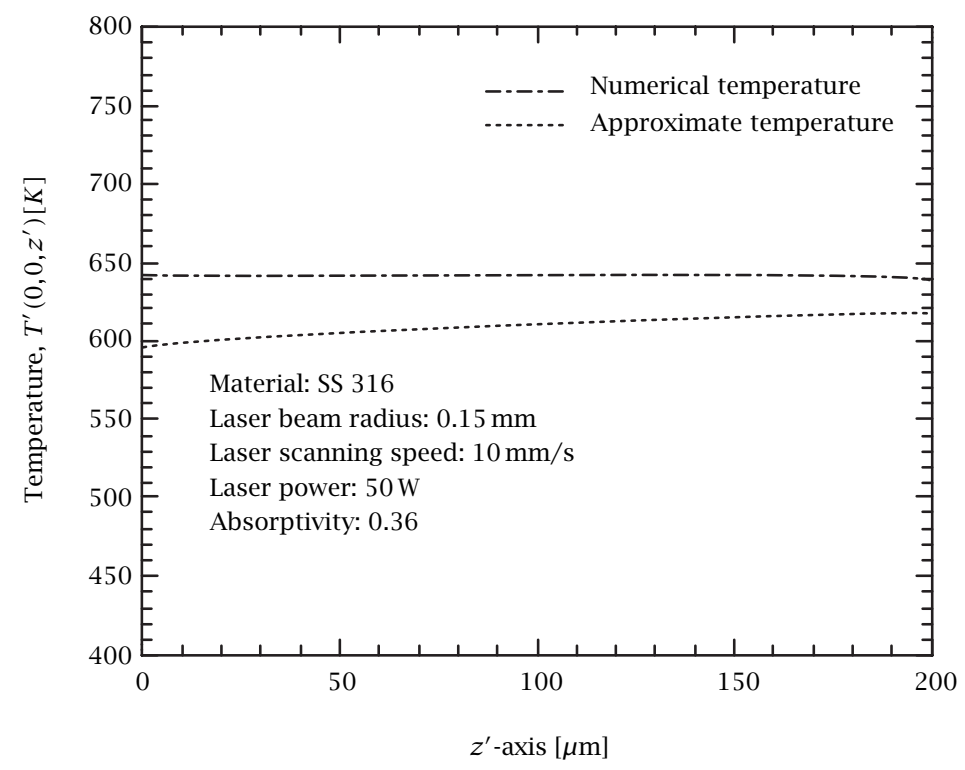

FIGURE 2.2. Comparison between the numerical and approximate temperatures $(r=0.45 \mathrm{~mm}$ case). 


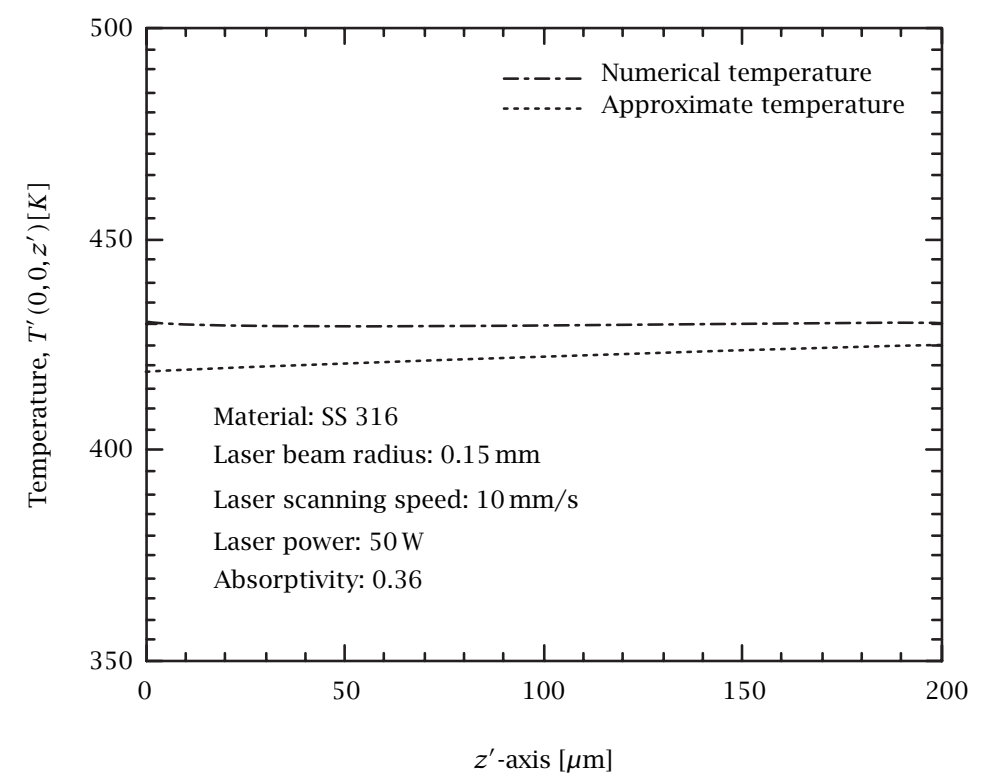

FIGURE 2.3. Comparison between the numerical and approximate temperatures $(r=0.75 \mathrm{~mm}$ case).

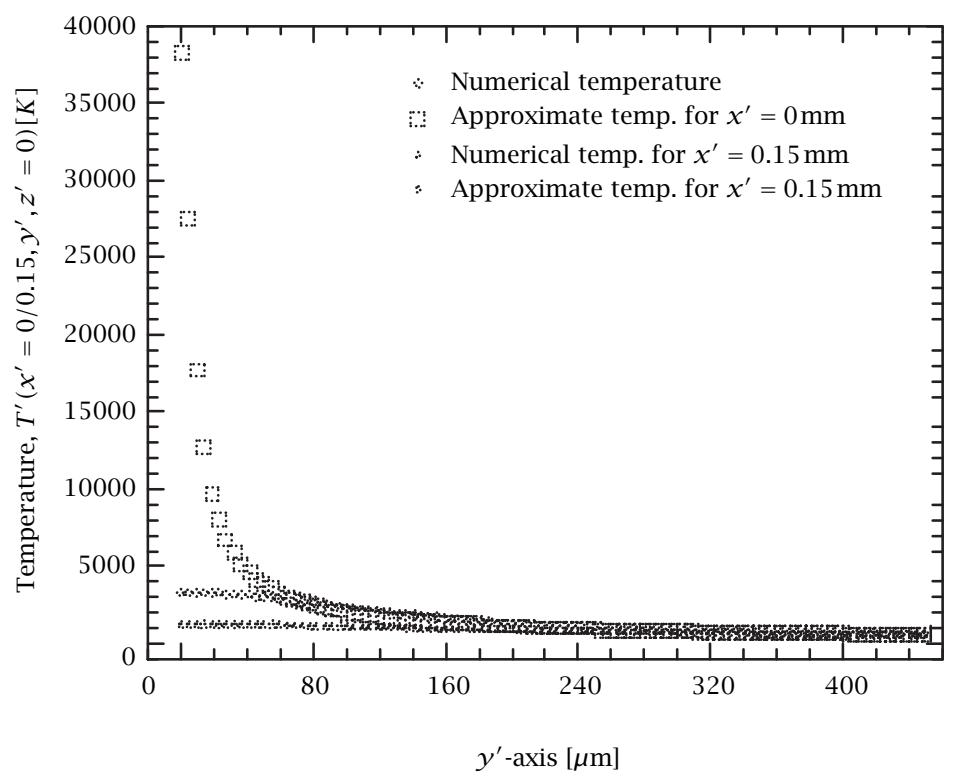

FIGURE 2.4. Comparison between the numerical and approximate temperatures in the $y^{\prime}$ direction for the different values of $x^{\prime}$. 


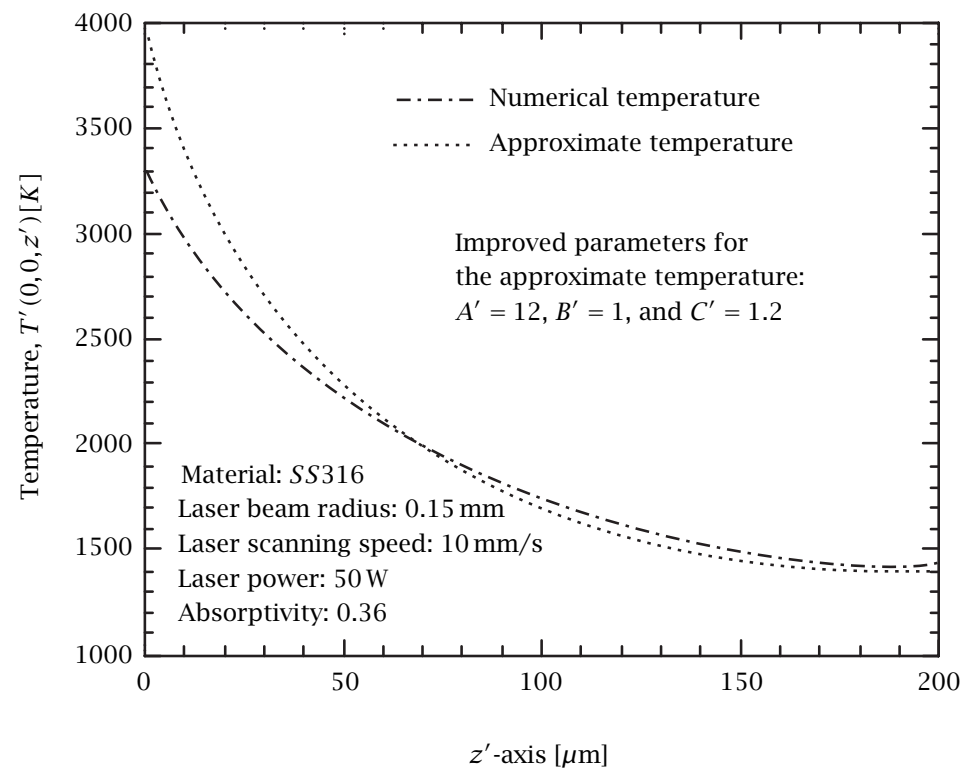

FIGURE 2.5. Comparison between the numerical and improved approximate temperatures ( $r=0$ case).

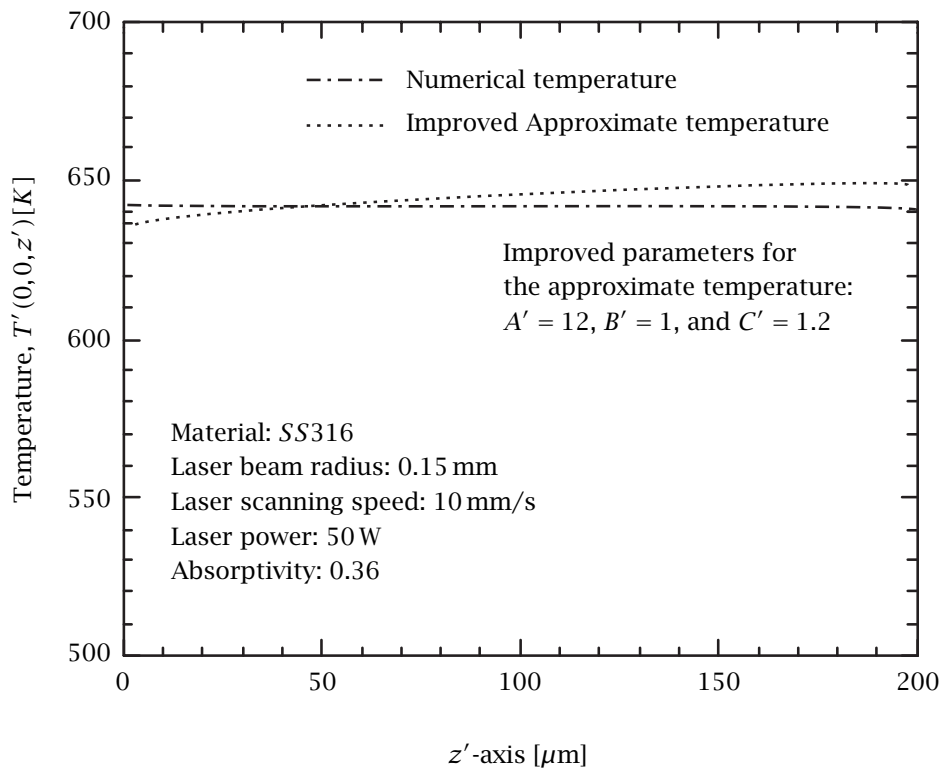

FIGURE 2.6. Comparison between the numerical and improved approximate temperatures ( $r=0.45 \mathrm{~mm}$ case). 


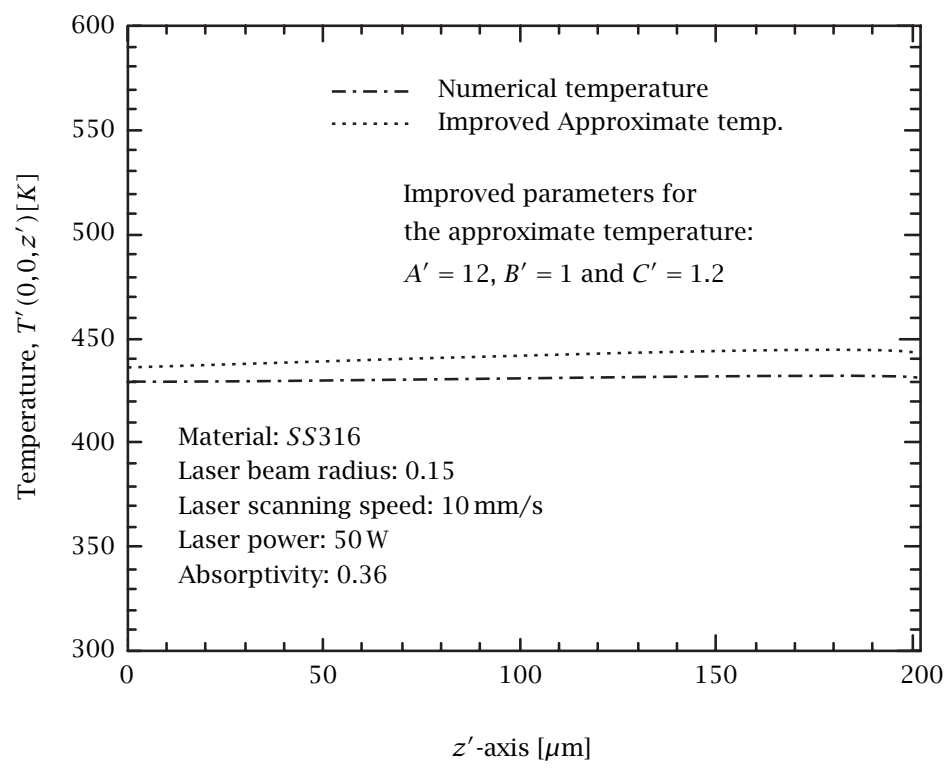

FIGURE 2.7. Comparison between the numerical and improved approximate temperatures ( $r=0.75 \mathrm{~mm}$ case).

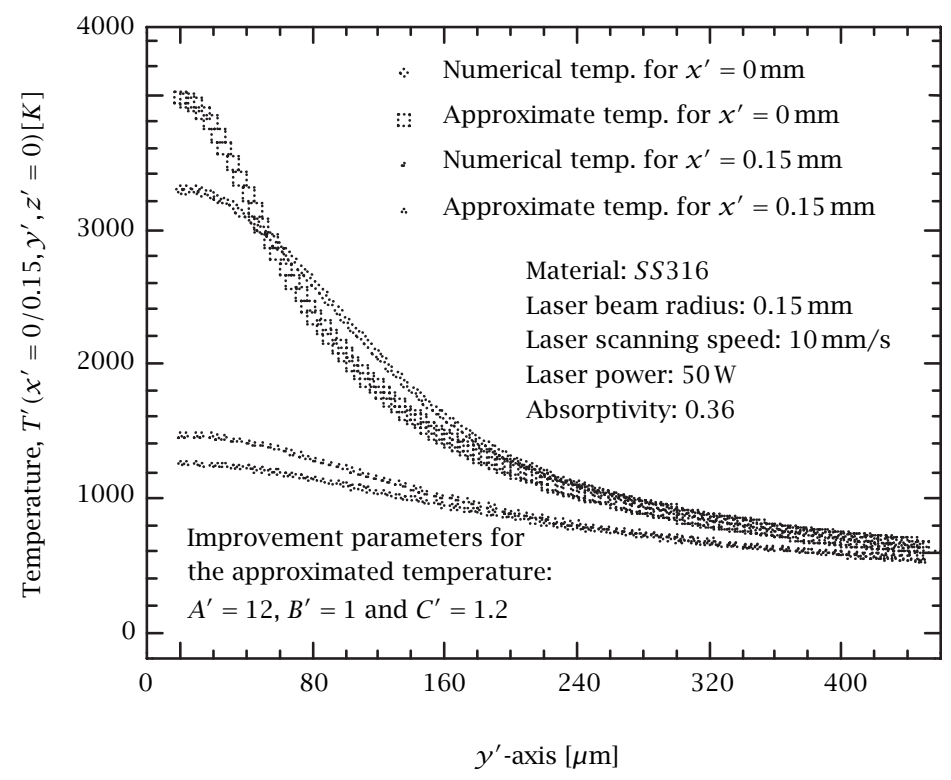

FIGURE 2.8. Comparison between the numerical and approximate temperatures in the $y^{\prime}$-direction for different values of $x^{\prime}$. 
3. Conclusion. The numerical and approximate temperature values are presented for different values of the radial distance $r$ in the real physical space. The approximation is based on the fact that the integral expression, (1.13), is accurate around $R=0$ which means larger values of $r$. To fix this problem, (2.1) is used for the optimal choices of the parameters $A^{\prime}=12, B^{\prime}=1$, and $C^{\prime}=1.2$. This approximation is also good for smaller values of $R$. The values of these parameters are determined numerically.

ACKNOWLEDGEMENTS. This project was sponsored by the US Air Force and Metal Tech Industries. We are grateful to Joe Longobardi, President of Metal Tech Industries, and Dr. W. P. Latham for all their support and helpful comments.

\section{REFERENCES}

[1] A. Erdélyi, W. Magnus, F. Oberhettinger, and F. G. Tricomi, Tables of Integral Transforms. Vol. II, McGraw-Hill, London, 1954, based, in part, on notes left by Harry Bateman. MR 16,468c. Zbl 058.34103.

[2] U. Tanriver, J. Longobardi, W. P. Latham, and A. Kar, Effects of absorptivity, shielding gas speed, and contact media on sheet metal laser welding, Science and Technology of Welding and Joining 5 (2000), no. 5, 310-316.

Ugur TANRIVER: SCHOOL OF OPTICS, CENTER FOR RESEARCH AND EDUCATION IN OPTICS AND LASERS (CREOL), MECHANICAL, MATERIALS AND AEROSPACE ENGINEERING DEPARTMENT, UNIVERSITY OF CENTRAL FLORIDA, ORLANDO, FL 32816, USA

ARAVINDA KAR: SCHOOl OF Optics, CENTER FOR RESEARCH AND EDUCATION IN OPTICS AND LASERS (CREOL), MECHANICAL, MATERIALS AND AEROSPACE ENGINEERING DEPARTMENT, UNIVERSITY OF CENTRAL FLORIDA, ORLANDO, FL 32816, USA

E-mail address: akar@mai 1.ucf.edu 


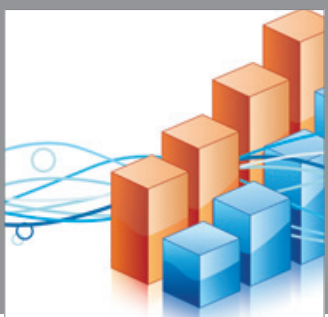

Advances in

Operations Research

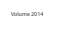

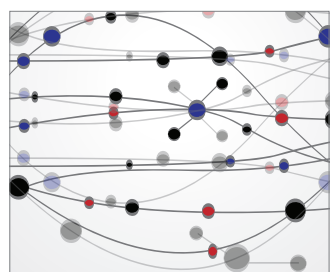

\section{The Scientific} World Journal
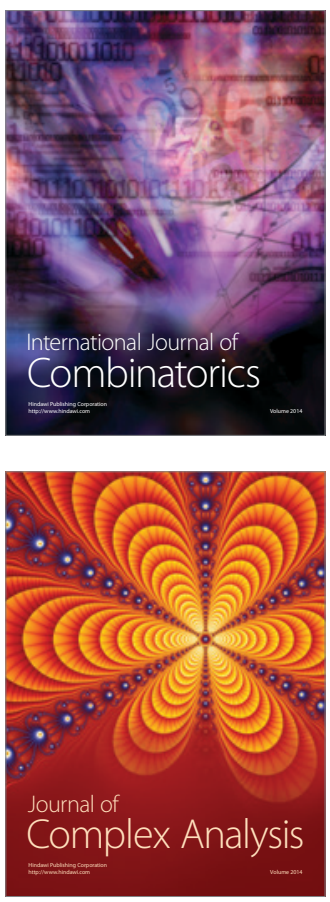

International Journal of

Mathematics and

Mathematical

Sciences
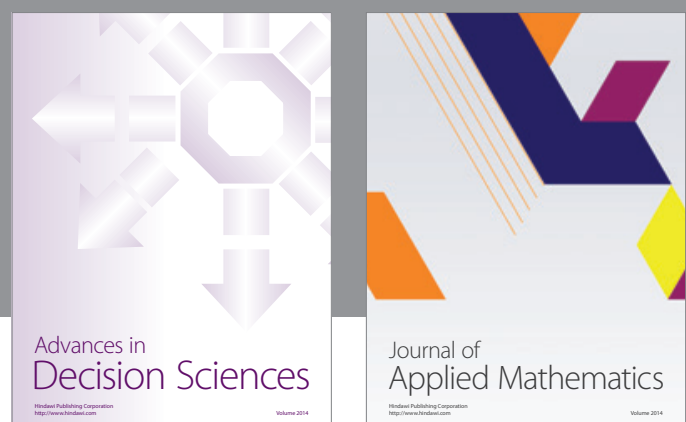

Journal of

Applied Mathematics
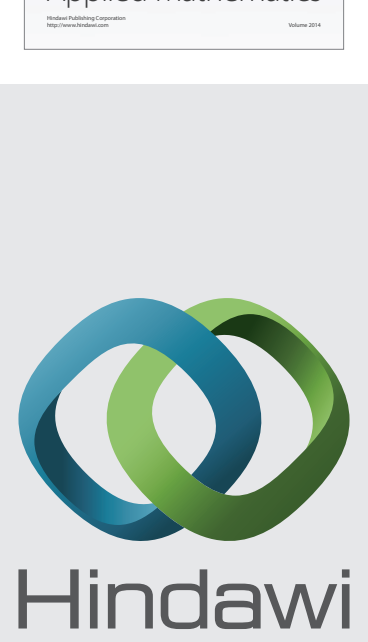

Submit your manuscripts at http://www.hindawi.com
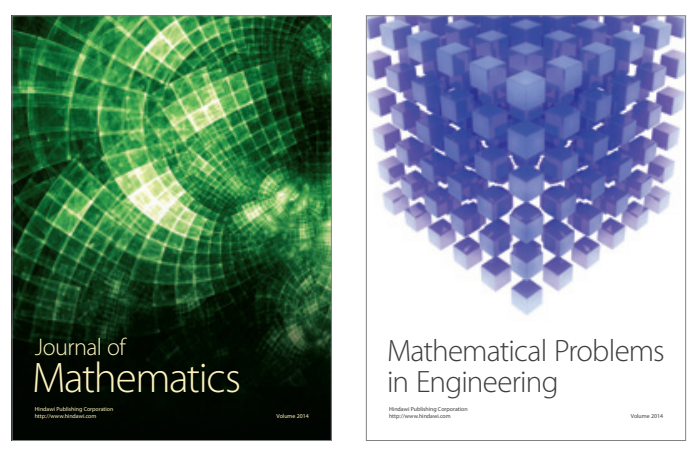

Mathematical Problems in Engineering
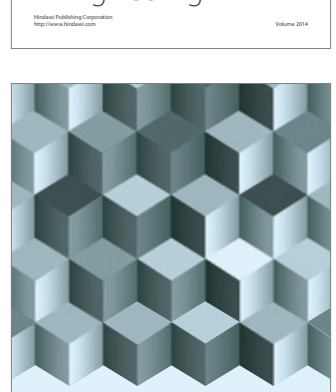

Journal of

Function Spaces
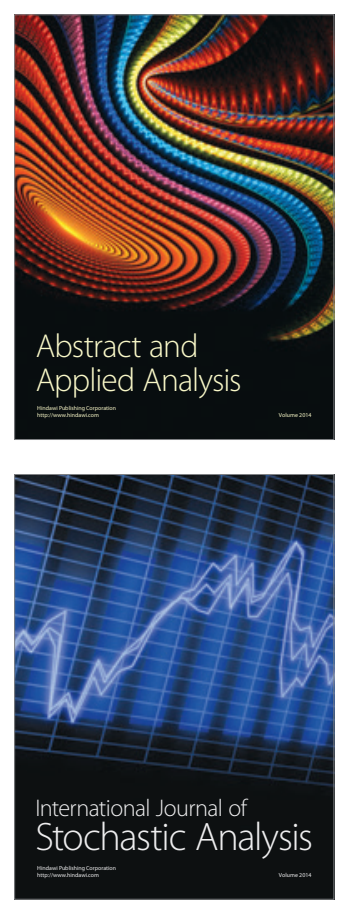

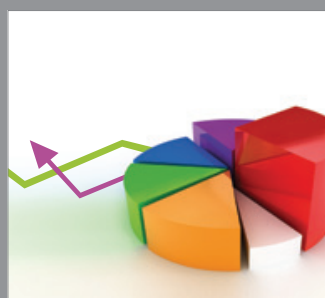

ournal of

Probability and Statistics

Promensencen
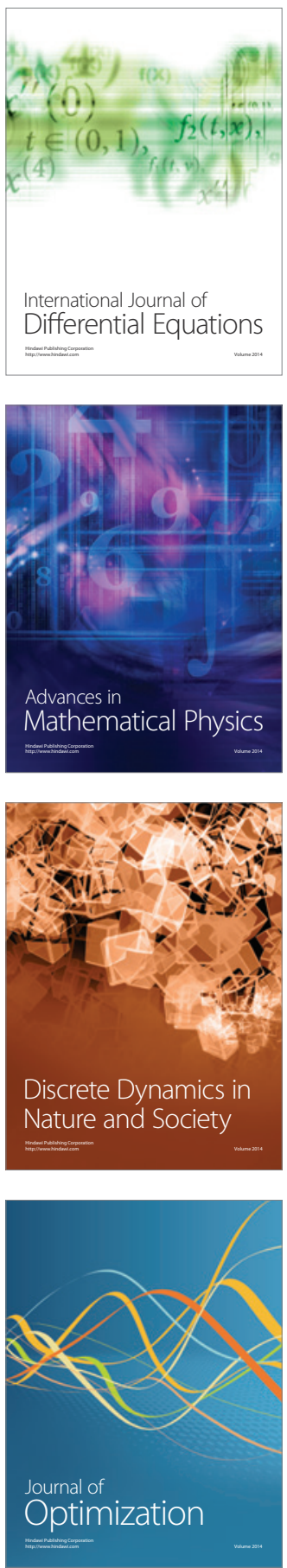